\title{
Multiple-Planet Scattering and the Origin of Hot Jupiters
}

\author{
C. Beaugé ${ }^{1,2}$ and D. Nesvorný ${ }^{2}$ \\ (1) Observatorio Astronómico, Universidad Nacional de Córdoba, \\ Laprida 854, X5000BGR Córdoba, Argentina \\ (2) Department of Space Studies, Southwest Research Institute, \\ 1050 Walnut St., Suite 300, Boulder, CO 80302, USA
}

Received __; accepted _ _ 


\begin{abstract}
Doppler and transit observations of exoplanets show a pile-up of Jupiter-size planets in orbits with a 3-day period. A fraction of these hot Jupiters have retrograde orbits with respect to the parent star's rotation, as evidenced by the measurements of the Rossiter-McLaughlin effect. To explain these observations we performed a series of numerical integrations of planet scattering followed by the tidal circularization and migration of planets that evolved into highly eccentric orbits. We considered planetary systems having 3 and 4 planets initially (before the scattering phase), located at 1-15 AU, and with masses between 0.5 and 4 times that of Jupiter. The simulations included the tidal and relativistic effects, and precession due to stellar oblateness. Stellar and planetary tides were modeled in the approximation of the time-lag equilibrium model that was modified for quasi-parabolic orbits to include the main features of the dynamical tide model. We found that the standard Kozai migration is an inefficient mechanism for the formation of hot Jupiters, as the orbits typically acquire high eccentricities and high inclinations due to close encounters and subsequent slow secular interactions, rather than due to the sole effect of the Kozai resonance.

Our results show the formation of two distinct populations of hot Jupiters. The inner population (Population I) of hot Jupiters with semimajor axis $a<$ $0.03 \mathrm{AU}$ formed in the systems where no planetary ejections occurred. This group contained a significant fraction of highly inclined and retrograde orbits, with distributions largely independent of the initial setup. However, our followup integrations showed that this populations was transient with most planets falling inside the Roche radius of the star in $<1$ Gyr. The outer population of hot Jupiters (Population II) formed in systems where at least one planet was ejected into interstellar space. This population survived the effects of tides over
\end{abstract}


$>1$ Gyr. The semimajor axis distribution of Population II fits nicely the observed 3-day pile-up.

A comparison between our 3-planet and 4-planet runs shows that the formation of hot Jupiters is more likely in systems with more initial planets. This appears related to an increase in the chaoticity of the system and to a larger number of close encounters. For example, the planetary systems with four initial planets produce hot Jupiters twice as often as those with three planets. Interestingly, the inclination distribution of Population II also depends on the number of planets in the initial systems. While we found only a few hot Jupiters in retrograde orbits in the 3-planet case, the 4-planet case showed a larger proportion (up to 10\%), and a wider spread in inclination values. As the later results roughly agrees with observations, this may suggest that the planetary systems with observed hot Jupiters were originally rich in the number of planets, some of which were ejected. In a broad perspective, our work therefore hints on an unexpected link between the hot Jupiters and recently discovered free floating planets.

Subject headings: planets and satellites: general, methods: N-body simulations 


\section{Introduction}

To date, the Rossiter-McLaughlin effect has been measured for 37 exoplanets (Moutou 2011). Of these, 8 planets $(\sim 20 \%)$ are in retrograde orbits, while in about half of the cases the planet orbit normal is probably aligned with the stellar spin vector $\left(|\lambda|<30^{\circ}\right.$, where $\lambda$ is the usual projected spin-orbit misalignment angle). Interestingly, the known planets with $|\lambda|>40^{\circ}$ have masses $M \lesssim 2 \mathrm{M}_{\mathrm{Jup}}$, where $\mathrm{M}_{\mathrm{Jup}}$ is the mass of Jupiter, while planets with $M>3 \mathrm{M}_{\text {Jup }}$ have $|\lambda| \gtrsim 40^{\circ}$ (Moutou 2011). If this trend holds with the new data, it could provide an important hint on the dynamical origin of the misaligned population. For example, as we will discuss in detail in Section 3, planet scattering followed by tidal circularization and migration is expected to produce such a trend because the less massive planets generally evolve into more eccentric and inclined orbits than the more massive ones, and are therefore more likely to show misalignment.

There also seems to be an indication that more circular orbits are accompanied by small values of $|\lambda|$, while large values of $|\lambda|$ appear in more eccentric cases (Schlaufman 2010). This can be interpreted as the evidence for two distinct populations of close-in exoplanet systems. The former population can be consistent with the smooth planetary migration in a gaseous disk (e.g., Benitez-Llambay et al. 2011), while the later probably requires a more complex orbital history.

To produce large values of $|\lambda|$, it is either necessary to tilt the spin axis of the star so that it ends up being misaligned with the original protoplanetary disk in which planets formed, or to tilt the planetary orbit. A tilt of the star's axis could be produced by the cumulative effect of stellar flares, late unisotropic (Bondi-Hoyle) accretion on the star (Throop \& Bally 2008, Moeckel \& Throop 2009), and due to the interaction between the stellar magnetic field and the protoplanetary disk (Lai et al. 2011, and the references therein). An orbital tilt can be produced by: (i) Planetary scattering followed Kozai 
migration (e.g. Nagasawa et al. 2008); (ii) Kozai migration produced by a planetary perturber in a distant and inclined orbit (Naoz et al. 2011); and (iii) Secular migration in well-spaced, eccentric, and inclined planetary systems (Wu \& Lithwick 2011) 1

Here we concentrate on the orbital tilt theories, because observational evidence suggests that at least some planetary systems have large orbital inclinations (e.g., planets $c$ and $d$ of $\nu$ Andromedae have mutual inclination $I \sim 30^{\circ}$; McArthur et al. 2010). In addition, the observed large eccentricities of exoplanets can be best explain if the original packed planetary systems underwent a dynamical instability followed by planet scattering (e.g., Weidenschilling \& Marzari 1996, Rasio \& Ford 1996). As planet scattering naturally leads to large orbital inclinations as well, the orbital tilt theories are therefore a logical extension of the generally accepted planet scattering model.

Theories (i) and (ii) listed above invoke the so-called Kozai mechanism, or Lidov-Kozai resonance (Lidov 1961, Kozai 1962), to drive up the orbital inclination $I$ towards values larger than ninety degrees. As the Lidov-Kozai resonance appears in the secular dynamics of the three-body problem for mutual inclinations $I \gtrsim 40^{\circ}$ (Libert \& Henrard 2007), a question arises of how such a large inclination between planetary orbits can be achieved in the first place. In addition, the retrograde orbits can be produced in the octupole (and higher order) Kozai approximation only for a relatively small subset of initial conditions. Naoz et al. (2011) did not address these issues in detail.

Wu \& Lithwick (2011) argued that the retrograde orbits can be achieved is systems with a significant initial Angular Momentum Deficit (AMD) and well separated planetary orbits. They showed that the smaller planets in these systems can exchange angular

\footnotetext{
${ }^{1}$ Inclined stellar perturber could in principle also trigger Kozai migration, but no distant star companions are observed in systems with known hot Jupiters.
} 
momentum with the more massive planets and evolve into highly-inclined, and possibly retrograde orbits, by the slow secular interaction between orbits. Therefore, this mechanism is similar to that discussed in Naoz et al. (2011), but can potentially be more efficient than the Kozai resonance in that it does not require finely-tuned initial conditions. Still, it remains to be explain how the large AMD assumed by Wu \& Lithwick (2011) arises in planetary systems as planets should form with a very low AMD.

The initial AMD can arise as a result of planet scattering, which brings us back to the statistical study of Nagasawa et al. (2008). Somewhat ironically, the initial setups of Naoz et al. (2011) and Wu \& Lithwick (2011) could therefore be traced to planet scattering (unless alternative explanations are offered for their initial conditions; e.g., Libert \& Tsiganis 2011ab).

Nagasawa et al. study was done before the first retrograde planets were discovered so that, understandingly, not much effort was invested in their work in a detail analysis of the mechanism that produced retrograde planets. This is where the works of Naoz et al. (2011) and $\mathrm{Wu} \&$ Lithwick (2011) come to be handy. As we discuss in detail in section 3, the Kozai mechanism alone appears to be inefficient in producing the retrograde hot Jupiters. Instead, we find that the orbital tilt is usually achieved through a combination of planet scattering, and the secular chaos of Wu \& Lithwick (2011).

While many works considered planet scattering (e.g. Marzari \& Weidenschilling 2002, Chatterjee et al. 2008, Juric \& Tremaine 2008), Nagasawa et al. (2008) pioneered the statistical studies of planet scattering with tidal effects. The principal role of tides is the circularization of the planetary orbit while approximately preserving the angular momentum. This effect helps to stabilize the orbits of scattered planets reaching small pericenter distances $(q \lesssim 0.05 \mathrm{AU})$ and, consequently, leads to the formation of hot-Jupiters.

For the tidal effects, Nagasawa et al. (2008) adopted the dynamic tide model by Ivanov 
\& Papaloizou (2004), which is applicable for fully convective planets with near-parabolic orbits, but is not well suited for low-to-moderate eccentricities. Conversely, Wu \& Lithwick (2011) used the equilibrium tide model (e.g. Hut 1981, Mardling 2007) which is strictly valid only for low-to-moderate eccentricities. Since the real evolution of planets likely spans the whole range from near-parabolic to near-circular orbits, it is not clear whether any of the two approximations mentioned above is adequate. We explain how we deal with this problem in Section 2.

For the work described in this paper we assumed a physical scenario similar to Nagasawa et al. (2008) starting with multiple-planetary systems in unstable orbits and following their evolution through the stage of close encounters and planet scattering. Our main simulations employed an N-body code, incorporating the relativistic effects, stellar oblateness and tidal precession. In Section 3 we describe our N-body code and the results of scattering experiments with 3-planet systems. An extension to 4 planets is reported, fittingly, in Section 4. The long-term tidal evolution of hot Jupiters is discussed in Section 5 .

\section{Tidal Model}

\subsection{Equilibrium vs. Dynamical Tides}

Current tidal models were constructed for two limit cases. If the orbital separation between the interacting bodies is roughly constant due to low orbital eccentricity, the tides vary slowly and generate an equilibrium figure in the extended bodies. Viscosity causes this tidal bulge to deviate from the instantaneous equipotential shape which, in turn, leads to an angular momentum exchange between the orbital and rotational motions. The dynamical evolution of the bodies in this approximation is described by the so-called equilibrium tide 
model, originally developed by Darwin (1879). In its simplest version (Mignard 1979, 1980), it is assumed that the equilibrium shape of each body at time $t$ is defined by the equipotential surface at time $t+\delta t$. The time lag $\delta t$ can be either positive or negative depending on whether the rotational period is larger or smaller than the orbital period.

The opposite limit case occurs when $e \sim 1$. In this case the tidal distortion is generated only at the pericenter and is negligible during the rest of the orbit. Consequently, the bodies can no longer achieve equilibrium figures. Instead, they undergo forced oscillations, the most important being f-mode (or surface gravity) waves. The subsequent effects on the rotational and orbital motion are described by the dynamical tide model (e.g. Lai 1997, Ivanov \& Papaloizou 2004, 2007, 2011). Dynamical tides are much more complex than their equilibrium counterparts and their effect on the orbital and rotational evolution of the participating bodies is not so well understood. For example, up to date only planar or polar orbits have been studied, and there is no model for planets with arbitrary inclinations.

Unfortunately, the dynamical evolution that leads to formation of hot Jupiters covers both limit cases discussed above. Initially, the planet has an almost parabolic orbit caused by scattering and slow secular evolutions. Thus, the tidal interaction with the star should be treated in the frame of the dynamical tide model. As the orbit decays and circularizes, the system approaches the equilibrium tide regime, and the dynamical tide model ceases to be valid.

Since the two models are based on a consideration of two completely different physical phenomena (equipotential figures vs. damped forced oscillations), it may be difficult to construct an unified physical tidal model that would be adequate for the entire orbital evolution of hot Jupiter. To deal with this problem we empirically modified the equations of the equilibrium tide model to mimic the effects of the dynamical tide model when the parabolic limit is approached. 


\subsection{Equilibrium Tidal Model}

We use the equilibrium tidal equations derived in Correia et al. (2011). These equations are based on the linear Mignard model, and contain explicit expressions for the variations of the mutual inclination, argument of the pericenter and obliquities, all in a consistent manner. They also include additional perturbations from gravitational interactions with other planets, stellar oblateness and general relativity in the post-Newtonian approximation.

Tidal precession is usually neglected in tidal equations, since the precessional period is much shorter than the timescale associated to circularization and orbital decay. However, since it is expected that Lidov-Kozai resonance may play an important role in the orbital evolution of hot Jupiters, we retain these secular terms in our model.

We introduce two important changes with respect to Correia et al. (2011). First, instead of limiting the gravitational interactions between the planets to the quadrupole secular approximation, we extend it to the octupole level. Second, instead of adopting Jacobi coordinates, we use Poincaré astrocentric coordinates.

We assume three extended bodies: $m_{0}$ (star), $m_{1}$ (inner planet) and $m_{2}$ (outer planet), with the planets having orbital elements $a_{i}, e_{i}, I_{i}$ and $\omega_{i}$, where $\omega_{i}$ are arguments of pericenters. Tidal effects will only be felt by $m_{0}$ and $m_{1}$. The outer body is assumed to be too far from the central star to generate or receive tidal distortions, but will interact gravitationally with $m_{1}$.

We use the following variables for the orbital motion:

$$
\begin{aligned}
\mathbf{G}_{\mathbf{i}} & =\beta_{i} \sqrt{\mu_{i} a_{i}\left(1-e_{1}^{2}\right)} \hat{\mathbf{k}}_{\mathbf{i}} \\
\mathbf{e}_{\mathbf{i}} & =\frac{\dot{\mathbf{r}}_{\mathbf{i}} \times \mathbf{G}_{\mathbf{i}}}{\beta_{i} \mu_{i}}-\frac{\mathbf{r}_{\mathbf{i}}}{r_{i}}
\end{aligned}
$$

where $\beta_{i}$ and $\mu_{i}$ are the Poincaré mass factors (e.g. Laskar \& Robutel 1995), $\mathbf{G}_{\mathbf{i}}$ are the orbital angular momentum vectors for each mass and $\mathbf{e}_{\mathbf{i}}$ the Lenz vectors. The unit vector 
$\hat{\mathbf{k}}_{\mathbf{i}}$ is perpendicular to the orbital plane (the reference plane is arbitrary), while the direction of the Lenz vector points towards the argument of pericenter.

Additionally, for the tidally interacting bodies, we also define the rotational frequencies by $\Omega_{0}$ and $\Omega_{1}$. The equations of motion for the objects' spins will be written in terms of the rotational angular momenta:

$$
\mathbf{L}_{\mathbf{i}}=C_{i} \Omega_{i} \hat{\mathbf{s}}_{\mathbf{i}}
$$

where $C_{i}$ are the principal moments of inertia and $\hat{\mathbf{s}}_{\mathbf{i}}$ the spin axis referred to the same reference plane. It is assumed that the spin vectors always coincide with the orientation of the principal moments of inertia.

Correia et al. (2011) constructed the variational equations for the complete set of variables $\left(\mathbf{G}_{\mathbf{i}}, \mathbf{e}_{\mathbf{i}}, \mathbf{L}_{\mathbf{i}}\right)$ that include all the above-mentioned perturbations, from secular terms of the mutual gravitational interactions between the planets to tidal effects, GR and stellar oblateness. At any given instant, the eccentricities can be obtained from the modulus of the Lenz vector, the semimajor axis from $\left|\mathbf{G}_{\mathbf{i}}\right|$ and the rotational frequencies from $\left|\mathbf{L}_{\mathbf{i}}\right|$. The mutual inclination of both orbits can be calculated from:

$$
\cos I_{\text {mut }}=\hat{\mathbf{k}}_{\mathbf{1}} \cdot \hat{\mathbf{k}}_{\mathbf{2}}
$$

Finally, the obliquities can be determined from:

$$
\cos \theta_{i}=\hat{\mathbf{s}}_{\mathbf{i}} \cdot \hat{\mathbf{k}}_{\mathbf{1}} \quad ; \quad \cos \varepsilon_{i}=\hat{\mathbf{s}}_{\mathbf{i}} \cdot \hat{\mathbf{k}}_{\mathbf{2}}
$$

where $\theta_{i}$ is the obliquity of $m_{i}$ with respect to the orbital plane of the inner body, while $\varepsilon_{i}$ are the obliquities with respect to the orbital plane of the outer companion. We will be most interested in variables $\theta_{0}$ and $\theta_{1}$.

The orbital/rotational equations of motion yield a complete description of the tidal and gravitational evolution of the system, valid as long as no resonances or close encounters 

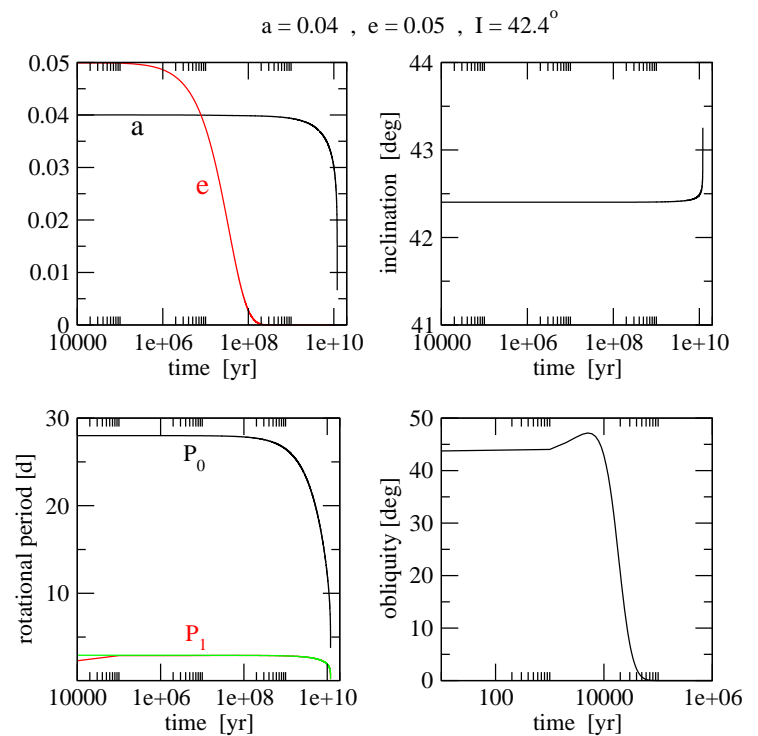

Fig. 1. - Tidal evolution of a Jovian mass planet with initial semimajor axis $a=0.04$ AU, $e=0.05$ and $I=45^{\circ}$ with respect to the stellar equator. $P_{0}$ is the stellar rotation period, while $P_{1}$ is the planet's rotation period. The green line in the bottom-left panel shows the planet's orbital period. The planet's obliquity shown in the bottom-right panel is measured with respect to the planetary orbital plane. The initial spin axis of the planet was assumed to coincide with that of the star.

between the planets are expected. It then constitutes a semi-analytical model that requires much less CPU time than a direct N-body integration.

Figures 1 and 2 show two examples, both with $m_{0}=M_{\odot}$ and $m_{1}=m_{1}=\mathrm{M}_{\mathrm{Jup}}$. For these illustrations, we used $Q_{*}^{\prime}=10^{7}$ and $Q_{p}^{\prime}=10^{5}$ for the star and planet tides, respectively. The quantity $Q^{\prime}=Q / k_{2}$ is the so-called modified tidal parameter, where $k_{2}$ is the tidal Love number. The value for the stellar parameter $Q_{*}^{\prime}$ was taken from Benitez-Llambay et al. (2011) as the value that best fits the semimajor axis distribution of short-period planets. The value for $Q_{p}^{\prime}$ follows from the recent determination for Jupiter (e.g. Lainey et al. 2009; but see Section 2.4 where we find $Q_{p}^{\prime}=(1-5) \times 10^{6}$ for hot Jupiters). 

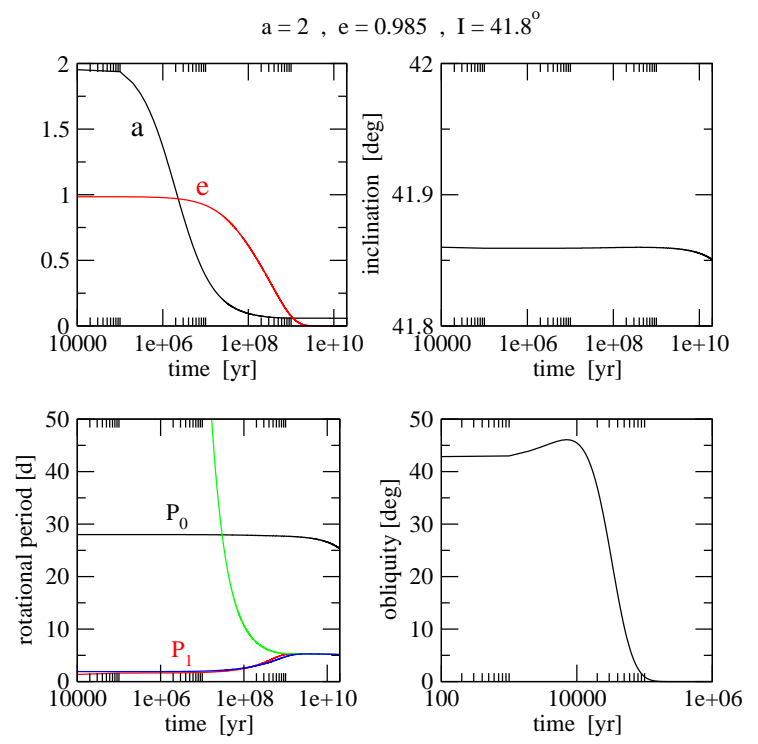

Fig. 2.- Same as Figure 1, but for an initial orbit with $e \sim 1$. The blue line in the bottom left panel shows $2 \pi / n_{q}$, where $n_{q}=\sqrt{\mathcal{G} / q^{3}}$.

In Fig. 1, the initial semimajor axes and eccentricity is similar to that of the known hot Jupiters $(a=0.04 \mathrm{AU}$ and $e=0.05)$. In Fig. 2, we assumed an initially quasi-parabolic orbit with larger semimajor axis $(a=2 \mathrm{AU}$ and $e=0.985)$ similar to those obtained from scattering experiments (e.g. Nagasawa et al. 2008). In the latter case, $q=1(1-e)=0.03 \mathrm{AU}$, thus placing the planet's pericenter within the region affected by tides. In both cases $m_{2}$ was placed in a distant circular orbit at $a_{2}=100$ AU to make its gravitational perturbations on the inner planet insignificant. The mutual inclination was chosen $I=45^{\circ}$, and both initial spin vectors were set to be perpendicular to the reference plane. Finally, the initial rotational periods were $P_{0}=2 \pi / \Omega_{0}=28$ days and $P_{1}=2 \pi / \Omega_{1}=0.4$ days.

Both simulations show practically no change in the orbital inclination due to tides, except during the final stages just before the planet is engulfed by the star. For quasi-circular orbits, the orbital decay time $\tau_{a}$ is longer than the circularization timescale $\tau_{e}$, implying 
that the planet is circularized before becoming a hot Jupiters (Fig. 1). The opposite occurs for the initially quasi-parabolic orbits, where $\tau_{e}>\tau_{a}$ (Fig. 2). This latter case is consistent with the results of Ivanov \& Papaloizou for dynamical tides (see also Nagasawa et al. 2008). However, even in this case, once the eccentricity decreases to small values, the relation switches back and the final stage of the orbital evolution occurs as in Figure 1,

Another difference in the high eccentricity case is that the synchronization of the planetary spin occurs with respect to the orbital frequency at pericenter and not with

respect to $n=\sqrt{\mathcal{G} / a^{3}}$. Once again, this is in good agreement with the predictions of Ivanov \& Papaloizou for the dynamical tide model. Thus, it appears that several of the orbital evolutionary properties of the dynamical tide model can be fairly and qualitatively reproduced with the equilibrium tide approximation.

Finally, we discuss the behavior of the planetary obliquity $\theta_{1}$. Planetary tides cause a relatively rapid alignment of the planet's spin axis with respect to the orbital plane normal, thus leading to $\theta_{1} \sim 0$. At least for the simulations discussed in this paper, we found no cases of trapping in other Cassini states. This occurs even for the retrograde orbits, where the initially obliquity was larger than $90^{\circ}$. However, the time scale of obliquity evolution is of the order of $10^{5}$ years. This is much larger than the synchronization timescale $\left(\sim 10^{4} \mathrm{yr}\right)$, but much smaller than the orbital decay times. Thus, in the absence of additional forces, it is expected that $\theta_{1} \sim 0$ during most of the planet's evolution.

\subsection{Correction Terms in the Tidal Model}

One of the most important differences between the equilibrium and dynamical tide models is their implication for the orbital decay and orbital circularization e-folding times ( $\tau_{a}$ and $\tau_{e}$, respectively). Figure 3 shows the value of $\tau_{a}$ obtained from the equilibrium 


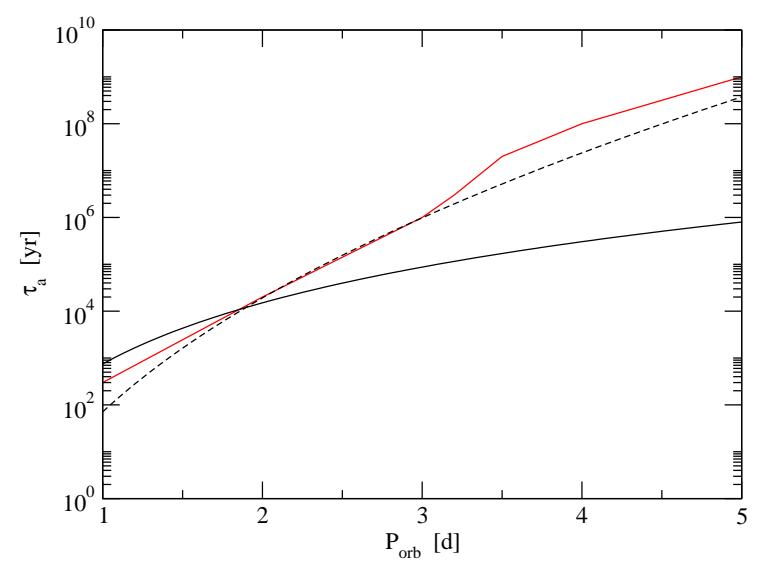

Fig. 3.- Orbital decay timescale as a function of the orbital period after circularization, for a Jovian mass planet in an initial quasi-parabolic orbit. Black curve is the prediction of the equilibrium tide model, while the red curve is the numerical estimate from the dynamical model from Ivanov \& Papaloizou (2011). The dashed curve was obtained from the equilibrium tide model by modifying the tidal parameters according to equation (5).

tide model (same tidal parameters as used in the previous figures) and from the dynamical model of Ivanov \& Papaloizou (2011) (see their Figure 5). The decay times are plotted as a function of $P_{\text {orb }}$ defined as the planet's orbital period after circularization. The dynamical model predicts shorter decay times for $P_{\text {orb }} \leq 2$ days and suggests much longer timescales for longer periods.

A way to reproduce, at least qualitatively, the results of Ivanov \& Papaloizou (2011) with the equilibrium tide model is to modify the values of the tidal parameters according to the following empirical recipe:

$$
Q_{i}^{\prime} \longrightarrow Q_{i}^{\prime} 10^{\beta} \text { with } \beta=2 e^{2}(a-3 / 2)
$$

The factor $e^{2}$ guarantees that this change is only significant for highly eccentric orbits, since for the lower eccentricity values the equilibrium tide model works well. Moreover, we implemented the correction term only for orbits with $a>1$ AU. This is because the 

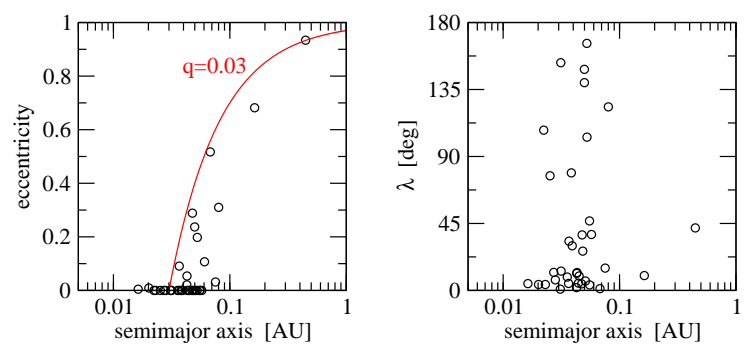

Fig. 4.- Distribution of known exoplanets with measured $\lambda$. Left plot shows eccentricity as a function of the semimajor axis. The red line denotes constant pericentric distance $q=0.03$ AU. Right plot shows $\lambda$ as function of the semimajor axis.

dynamical tide is expected to be relevant in our simulations only for large semimajor values.

The value of $\tau_{a}$ determined with this new tidal parameter is also plotted in Figure 3 , The overall agreement is satisfactory. The correction in Eq. [5 also affects the circularization timescale $\tau_{e}$, yielding values very close to those predicted by Ivanov \& Papaloizou (2011).

\subsection{Constraint on $Q_{p}^{\prime}$}

Figure 4 shows the distribution of known exoplanets with measured $\lambda$. Interestingly, the eccentricities show a marked correlation with the semimajor axis, roughly along a line with $q=0.03$ AU. This could potentially be used to constraint the value of $Q_{p}^{\prime}$.

To do that, we followed the tidal evolution of Jovian-mass planets. Their initial semimajor axis was taken from a uniform distribution in $\log (a)$ from 0.01 AU to 4 AU. The eccentricities were taken from a uniformly random distribution of $e$ between zero and one. A total of $10^{4}$ initial orbits were generated. Each orbit was evolved for 1 Gyr using our hybrid model. Results are shown in Figure 5 for four different values of the planetary tidal parameter $Q_{p}^{\prime}$, ranging from $10^{5}$ (top left) to $10^{7}$ (bottom right).

For $Q_{p}^{\prime}=10^{5}$, the envelope of final orbits does not seem to reproduce the observed 

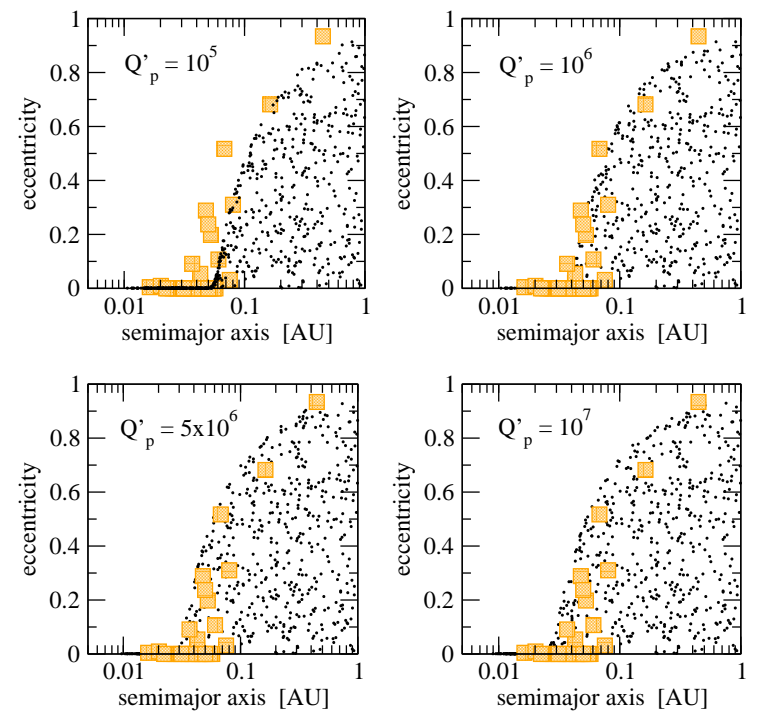

Fig. 5.- Eccentricity-semimajor axis distribution expected from tidal models with four different values of $Q_{p}^{\prime}$. Orange squares show the distribution of real exoplanets with measured $\lambda$ (data from www.exoplanet.eu webpage).

eccentricity distribution. The tidal effects are apparently too efficient, and all orbits with final semimajor axes below 0.06 AU become circularized. Better results are obtained with $Q_{p}^{\prime}=10^{6}$ and $Q_{p}^{\prime}=5 \times 10^{6}$, although larger values seem to be too inefficient.

If the real exoplanets are the outcome of scattering events and tidal capture from quasi-parabolic orbits, it is expected that they underwent a phase when their orbital eccentricities were were very high. Figure 6 factors this assumption in the results of our tests. Once again the standard equilibrium tide model with $Q_{p}^{\prime}=10^{5}$ does not yield a good agreement, but now it is clear that the modified model with $Q_{p}^{\prime}=10^{7}$ is also not adequate, since practically all the real planets fall into the region with relatively small initial eccentricities.

From these tests it appears that the eccentricity-semimajor axis distribution of the real exoplanets can be reproduced by planetary tidal parameters of the order of $Q_{p}^{\prime} \sim(1-5) \times 10^{6}$. 

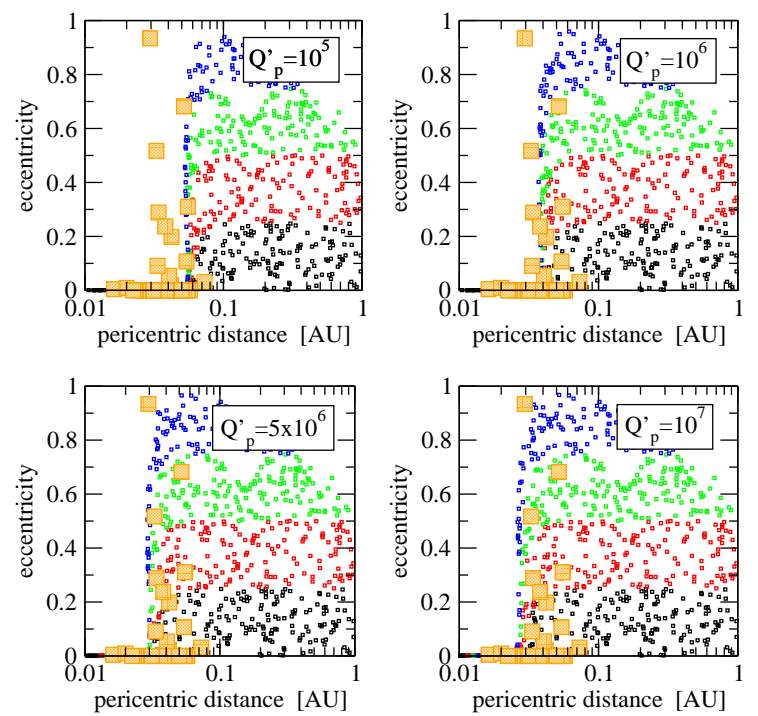

Fig. 6.- As Figure 5, but this time showing the final eccentricity in terms of the final pericentric distance. The color code identifies the initial eccentricity of each planet: black for those initial conditions with $e<0.25$, red for $0.25<e<0.5$, green for $0.5<e<0.75$ and blue for $e>0.75$. Th orbits of real exoplanets are labeled by orange squares.

Similar values have been proposed by other authors. Using similar analysis, Jackson et al. (2008) found that the distribution of all close-in planets shows good agreement assuming $Q_{p}^{\prime} \simeq 3 \times 10^{6}$, while a value of $Q_{p}^{\prime} \simeq 2.2 \times 10^{6}$ has recently been proposed to explain the current orbital characteristics of CoRoT-20b (Deleuil et al. 2011).

For the present work we mainly used $Q_{p}^{\prime}=5 \times 10^{6}$, and tested a range of $Q_{p}^{\prime}$ values in Section 3, and $Q_{*}^{\prime}=10^{7}$. As long as the planet has a non-negligible orbital eccentricity, the stellar tidal parameter $Q_{*}^{\prime}$ is of secondary importance because the orbital dynamics is mainly controlled by the planetary tide. However, the stellar tide is important once the planet reaches a circular orbit. 


\section{N-Body Simulations of 3-Planet Scattering}

To study the formation of hot planets, we must first follow the orbital evolution of a planetary system through the instability phase, when planets scatter off of each other. The code must also be able to track the evolution in the late stage when hot Jupiters become circularized and migrate by tides, as discussed in Section 2. Here we describe our N-body integrator that is able to handle close encounters between planets, near-parabolic

orbits that may result from encounters, and tides. To start with, we will apply this code to 3-planet systems, a case similar to that studied by Nagasawa et al. (2008).

\subsection{Explicit Expressions for the Forces}

In an astrocentric reference frame, the differential equation affecting the position vector $\mathbf{r}$ of the planet is:

$$
\ddot{\mathbf{r}}=\mathbf{f}_{0}+\mathbf{f}_{T P}+\mathbf{f}_{T D}+\mathbf{f}_{G R}+\mathbf{f}_{S O},
$$

where

$$
\mathbf{f}_{0}=-\frac{\mu}{r^{3}} \mathbf{r}
$$

is the gravitational acceleration from the central star, and $\mu=\mathcal{G}\left(m_{0}+m_{1}\right)$. The tidal distortion that generates the apsidal precession (see Hut 1981) is given by

$$
\mathbf{f}_{T P}=-3 \frac{\mu}{r^{8}}\left[k_{20}\left(\frac{m_{1}}{m_{0}}\right) R_{0}^{5}+k_{21}\left(\frac{m_{0}}{m_{1}}\right) R_{1}^{5}\right] \mathbf{r}
$$

while the corresponding tidal dissipation term is

$$
\begin{aligned}
\mathbf{f}_{T D}= & -3 \frac{\mu}{r^{10}} \sum_{i=0,1} \frac{m_{1-i}}{m_{i}} k_{2 i} \Delta_{i} R_{i}^{5} \\
& \left(2 \mathbf{r}(\mathbf{r} \cdot \dot{\mathbf{r}})+r^{2}\left(\mathbf{r} \times \boldsymbol{\Omega}_{i}+\dot{\mathbf{r}}\right)\right) .
\end{aligned}
$$

Here $\Omega_{i}$ are the spin vectors of both bodies with respect to the reference frame that does not have to coincide with the Laplace plane. The factor $k_{2 i} \Delta_{i}$ is related to the tidal parameter 
through:

$$
k_{2 i} \Delta_{i}=\frac{3}{2 Q_{i}^{\prime} n}
$$

where $n=\sqrt{\mu / a^{3}}$ is the mean motion of the planet. We used the hybrid equilibrium tidal model discussed in the previous section with $Q_{*}^{\prime}=10^{7}$ and $Q_{p}^{\prime}=5 \times 10^{6}$.

Finally,

$$
\mathbf{f}_{G R}=-\frac{\mu^{2} a\left(1-e^{2}\right)}{c^{2} r^{5}} \mathbf{r}
$$

where $c$ is the speed of light, is the post-Newtonian radial term that approximates the General Relativity effects, and

$$
\mathbf{f}_{S O}=\nabla\left(\mu J_{2} \frac{R_{0}^{2}}{r^{3}}\left(\frac{3}{2} \sin \delta-\frac{1}{2}\right)\right)
$$

is the acceleration from stellar oblateness (e.g. Beutler 2005), where $R_{0}$ is the stellar radius, $\delta$ the declination angle of the orbit with respect to the stellar equator, and $J_{2}$ the quadrupole coefficient.

From the complete expression for the acceleration we can calculate its effect on the spins, assuming a conservation of the complete (orbital + rotational) angular momentum (see Correia et al. 2011 for more details). We disregard any variation of the stellar rotational frequency due to momentum loss driven by stellar winds (e.g., Skumanich 1972), and assume that it is only affected by tides.

\subsection{The Code}

Our original plan was to implement these forces into SyMBA (Duncan et al. 1998), which is an efficient symplectic $N$-body code that is capable of tracking close encounters between massive bodies. As Mercury (Chambers 1999), SyMBA uses the Poincaré variables to be able to handle encounters. The symplectic algorithms written in Poincaré variables, 
however, have troubles in following orbits with very high eccentricities. This is a problem, because the orbits of hot Jupiters are expected to have $e \sim 1$ before they can become circularized by tides.

To solve this problem, Levison \& Duncan (2000) proposed a hybrid integration scheme in which the outer part of the eccentric planetary orbit is integrated with the usual $S y M B A$ algorithm. The code then symplectically switches to the Bulirsch-Stoer (BS) algorithm to follow the planet's evolution near the inner part of its orbit. The switch radius is set to a fixed apocentric distance, usually of order of $0.1 \mathrm{AU}$.

We tested the hybrid algorithm in the extreme case when $e \sim 1$ and found, perhaps not surprisingly, that the time-step needs to be set to a very small fraction of the orbital period. This slows down the symplectic algorithm so much that the Bulirsch-Stoer (BS) integrator, if used to follow the full evolution of the system (i.e., also outside the switch radius), is actually faster and more precise. For this reason, we constructed a new $N$-body code based on the BS method and used it in this study.

The code follows the interaction of $N$ massive planets orbiting a central star of mass $m_{0}$. It tracks both the orbital and spin dynamics according to the equations given in Section 3.1. As in Nagasawa et al. (2008), the integrations were stopped when reaching $10^{8}$ years, or if:

- A hot Jupiter formed with $e<0.01$ and stable orbit.

- One planet was ejected and the other two remained in stable orbits. In this case, the evolution of the system was continued using the secular model described in Section 2. The use of the semi-analytical model helped to speed up the simulation, and proved adequate for two-planet systems where no additional close encounters between planets occurred. 
- Two planets were ejected and the pericentric distance of the survivor was larger than 0.1 AU.

To determine whether a surviving pair of planets attained stable orbits we used the Hill criterion of Marchal \& Bozis (1982) (see also Gladman 1993).

\subsection{Initial Conditions}

Planetary migration produced by planet-gas-disk interactions is an important evolutionary process during the early history of planetary systems. As we do not model these early stages here, we will need, in an uncertain leap of faith, to adopt some initial conditions for our simulations. These condition should be at least broadly consistent with the state of the planetary systems just after the gas disk dispersal.

While classical hydrodynamical simulations of disk-planet interactions and resonance trapping have focused on two-planet systems (e.g. Snellgrove et al. 2001, Kley 2003), recent studied have been extended to multiple planetary systems (e.g. Morbidelli et al. 2007, Libert \& Tsiganis 2011a, 2011b). It appears that multiple-resonance trapping is a common outcome, although not all the resonant configurations are long-term stable. Thommes et al. (2008) also suggested that stable configurations within the gas disk may become unstable after disk dispersal and subsequent planetary scattering may occur.

We used several different mass ratios between planets. The masses in the units of $m_{\text {Jup }}$ were:

$$
\begin{aligned}
m_{1} & =1 \\
m_{(i+1)} & =H m_{i} \quad(i=2,3)
\end{aligned}
$$

where $H=0.5,1$ or 2 . After the mass values were specified (using a random generator for 
the $H$ values), we shuffled the radial order of the planets, such that the body identified as $m_{1}$ was not necessarily the one closest to the star.

The semimajor axis of the inner planet was chosen randomly between 1 and $5 \mathrm{AU}$. The other planets were placed in successive mean-motion resonances. We used the $2 / 1$, $3 / 2$ or $4 / 3$ resonant ratios for different planet pairs, with the specific choice depending on the individual masses of the two planets. To select the resonant ratio that should apply to a specific mass ratio, we adopted the results of Pierens \& Nelson (2008), who studied the resonant capture with a hydrocode. Using the same disk parameters as Pierens \& Nelson (2008), we also performed additional hydrocode simulations with FARGO (Masset 2000) to confirm and extend these results to multi-planet systems.

Orbital eccentricities were chosen randomly between zero and 0.1 and inclinations between zero and 1 degree. Although this seems arbitrary, we performed tests with different distributions (e.g. lower eccentricities for more massive planets) and found that the results were largely independent of these assumptions. The angular variables were randomly changed from their resonant values to mimic the situation at the onset of instability. All initial conditions were consequently dynamically unstable and none remained in the initial resonant configuration.

\subsection{Results}

In total, we followed the evolution of 2464 initial systems, a number sufficiently large for a detailed statistical analysis. Figure 7 shows the orbital distribution of all planetary systems at the end of simulations. A total of 288 hot Jupiters formed, which is approximately $11 \%$ of the number of initial systems. This fraction is about three times smaller than what was reported in Nagasawa et al. (2008). Most hot Jupiters acquired 

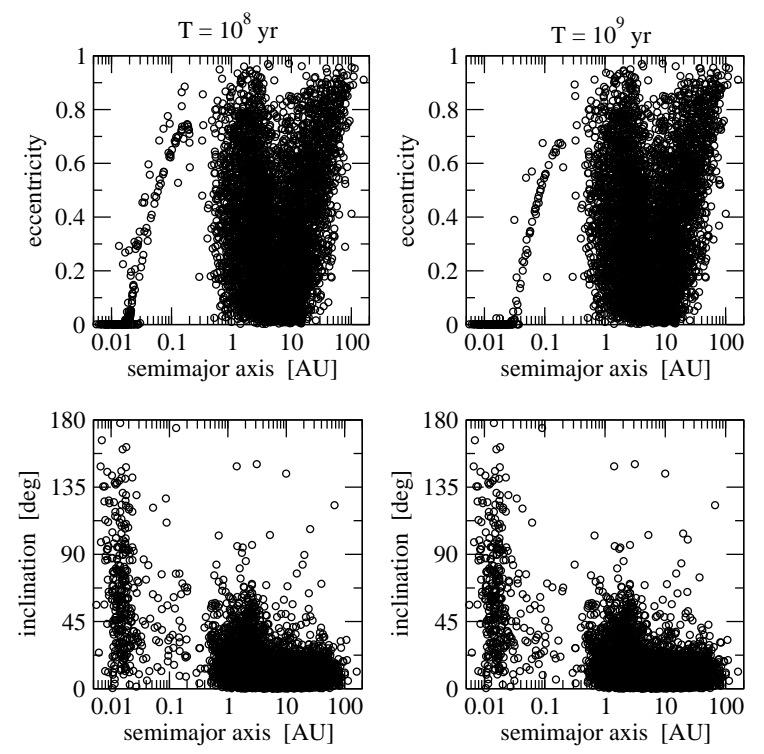

Fig. 7.- Left: Results of our 2464 N-body integrations of the 3-planet systems with $Q_{*}^{\prime}=10^{7}$ and $Q_{p}^{\prime}=5 \times 10^{6}$. The total integration time was set to $T=10^{8}$ yrs. Right: Extension to $T=10^{9}$ yrs using the semi-analytical model. We stopped the simulation if hot Jupiter formed and acquired a nearly circular orbit (defined as $e<0.01$ ).

circular orbits due to tidal damping. Some hot Jupiter, particularly those with $a \gtrsim 0.03$ AU, retained high eccentricities.

The left-hand panels in Figure 7 show the results of the $N$-body simulation for a total integration time of $T=10^{8}$ yrs. Since most of the known hot Jupiters with measured $\lambda$ have stellar ages of the order of 1 Gyr (e.g. Triaud 2011), we extended the simulations to see how the population of hot Jupiter can be modified by tidal effects over Gyr-long time scales. First, we selected the planetary systems, where the $N$ body integrations described above led to the formation of hot Jupiter. We disregarded the outer planets in each system because their interaction with the hot Jupiter is weak. We then used the semi-analytical code described in Section 2 to follow the tidal evolution of hot Jupiters up to $T=1$ Gyr. The results are shown in the right-hand plots of Figure 7. 

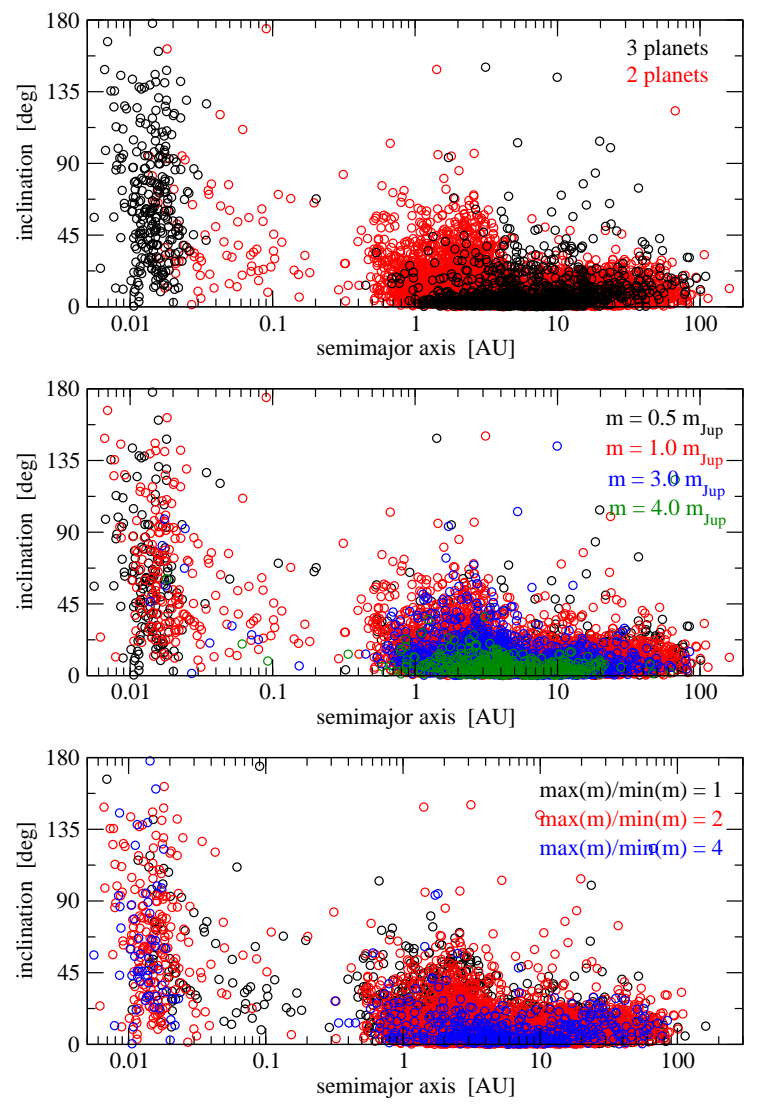

Fig. 8. - Distribution of the final orbital inclination as function of the semimajor axis. Top: Black circles indicate the planetary systems where no ejections occurred, while red symbols show those in which one planet was ejected. Middle: Color indicates the mass of each planet (see inlaid color code caption for details). Bottom: Color indicates the planet mass ratio defined as the ratio between the largest and smallest planet masses in the original systems.

Note that we stopped the simulation if a hot Jupiter evolved into a nearly circular orbit (defined as $e<0.01$ ). The results shown in Figure 7 therefore include all hot Jupiters that formed in our simulations at any time. As we will discuss in Section 3.6 and 4, many of these hot Jupiters evolve by tides and are dynamically short lived.

As for the eccentricity distribution, we find two types of final orbits. The first type consists of planets that where tidally trapped early in the simulations and had sufficient 
time to undergo tidal circularization. They show up in Fig. 7 as having nearly circular orbits. The second orbit type shows moderate to large eccentricities (in some cases as high as $e \sim 0.8$ ), and a clear correlation between $a$ and $e$. Such a correlation is expected for a population of planets evolving from quasi-parabolic orbits.

As for the inclination distribution, we note two distinct populations of hot Jupiters that can be conveniently classified as having $a \leq 0.03$ AU (Population I or Pop I) and $a>0.03 \mathrm{AU}$ (Pop II). Practically all orbits with $a>0.03$ AU have $I<90^{\circ}$, while about $22 \%$ of those with $a<0.03 \mathrm{AU}$ are retrograde.

A second difference between Pop I and Pop II is the behavior of planet's obliquity. Practically all Pop-I planets have zero obliquities (relative to planet's orbit), as expected from a sustained tidal evolution. In contrast, many Pop-II planets retained relatively large values of $\theta_{1}$. These planets continue to evolve by tides even 1 Gyr after their parent system's formation.

Next we studied the effect of planet masses and starting semimajor axes on the results (Figure 8). We find that Population I mainly contains planets that formed through scattering in systems in which no planet was ejected. The Pop-II planets, on the other hand, formed in systems where one planet was ejected. We discuss this interesting result in more detail in Section 3.5.

The middle frame in Figure 8 shows the $a$ and $I$ distribution sorted according to the planetary mass. This plot shows that the more massive planets remain near their original orbits at $a>1 \mathrm{AU}$. The hot Jupiters, on the other hand, tend to be the least massive planets in the original systems. This is easy to understand because the lighter planets are easier to scatter, and more likely to become hot Jupiters. Although there seems to be no significant difference in the inclination distribution between $m=0.5 \mathrm{M}_{\mathrm{Jup}}$ and $m=1 \mathrm{M}_{\mathrm{Jup}}$, the inclinations attained by the more massive planets are noticeably smaller. 

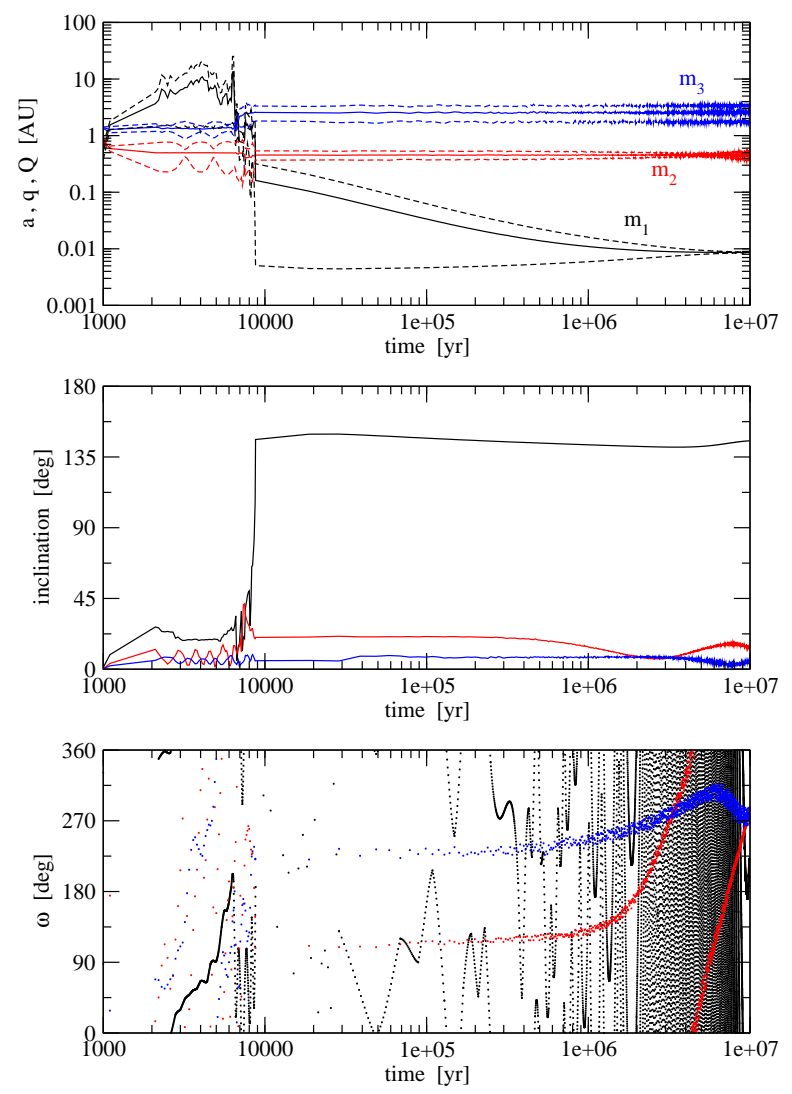

Fig. 9.- Orbital evolution of a 3-planet system leading to the formation of hot Jupiter on a retrograde orbit. Top: semimajor axis and pericentric/apocentric distance. Middle: orbital inclinations. Bottom: Pericenter arguments. Planetary masses were chosen equal to $m_{1}=1 \mathrm{M}_{\mathrm{Jup}}, m_{2}=2 \mathrm{M}_{\mathrm{Jup}}$ and $m_{3}=4 \mathrm{M}_{\mathrm{Jup}}$.

The bottom panel in Figure 8 separates the final planets according to the planet mass ratio in the initial systems. The results indicate that the Pop-I planets tend to form in planetary systems with a large mass ratio, while the outer Pop-II planets primarily evolve from the systems with planets more similar in mass. 

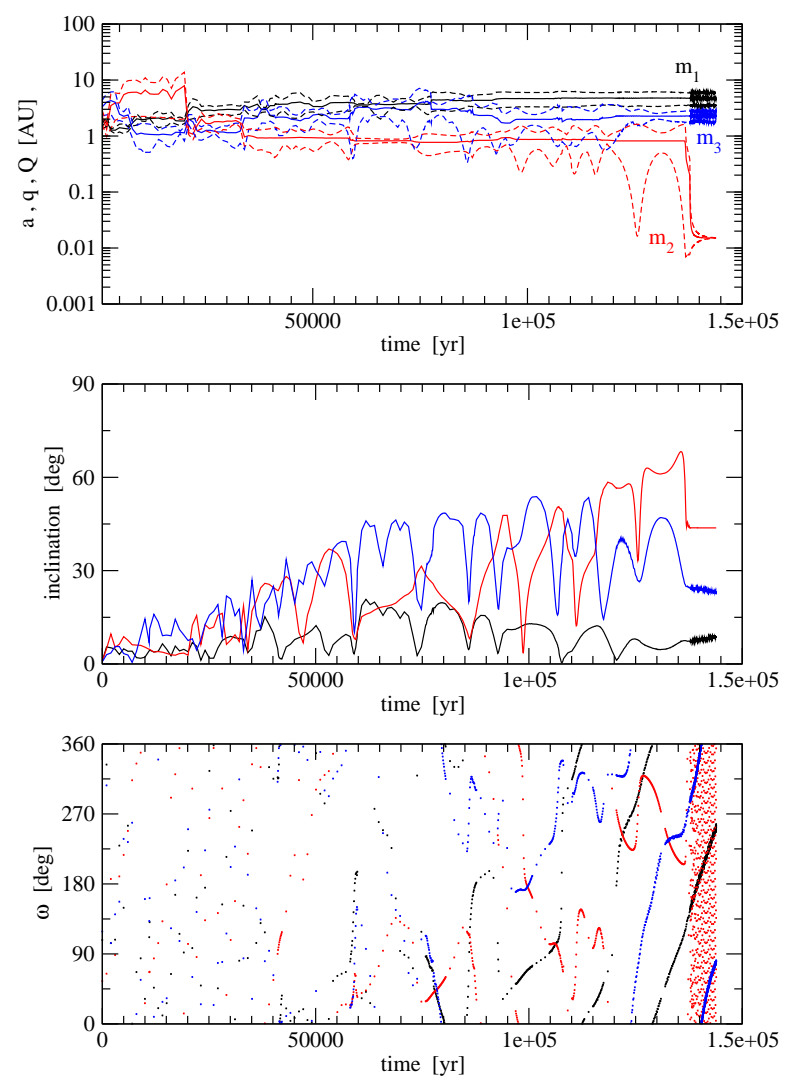

Fig. 10.- As Figure 9, but for a system that produced hot Jupiter on a prograde orbit. Note that the scale of the middle plot has changed with respect to Figure 9, Also, in this case, planetary masses were $m_{1}=1 \mathrm{M}_{\mathrm{Jup}}, m_{2}=0.5 \mathrm{M}_{\mathrm{Jup}}$, and $m_{3}=0.5 \mathrm{M}_{\mathrm{Jup}}$.

\subsection{Orbit evolution}

To understand the dynamical mechanism responsible for the formation of hot Jupiters, we now discuss the dynamical evolution of individual systems. We illustrate things on three different planetary systems.

Figure 9 shows the first case. In this case, none of the planets escapes, which is characteristic for the systems in which Pop-I hot Jupiters formed in our simulations. The initially inner planet $\left(m_{1}\right.$ shown in black) is quickly scattered to an exterior orbit. At $t \simeq 7000 \mathrm{yrs}$, it suffers a sequence of close encounters with the other two (more-massive) 

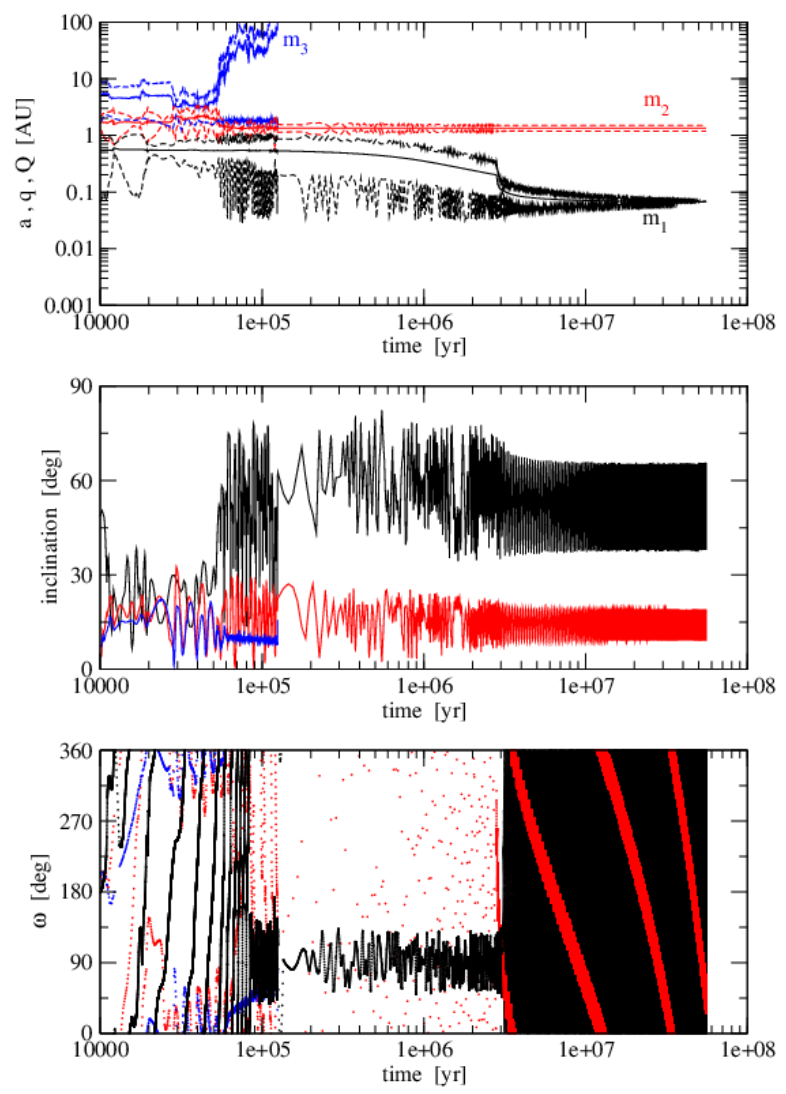

Fig. 11. - Example of a rare planetary system showing clear effects of the Lidov-Kozai resonance. Pop-II hot Jupiter formed in this simulation. Planetary masses were $m_{1}=1 \mathrm{M}_{\text {Jup }}$, $m_{2}=2 \mathrm{M}_{\mathrm{Jup}}$, and $m_{3}=1 \mathrm{M}_{\mathrm{Jup}}$.

planets, a period that lasts several thousand years. In consequence, the lighter planet is scattered into an inner orbit, its eccentricity raises to $\sim 1$, and orbital inclination very rapidly reaches values larger than $90^{\circ}$.

In the process, the pericentric distance drops and the planet decouples from other planets in the system to evolve solely by tides over the rest of its lifetime. Both the semimajor axis and eccentricity decrease due to tides while maintaining similar values of the pericentric distance (see lower black dashed curve in top panel of Figure 9). During this late stage, the inclination remains nearly constant and close the value excited by close 
encounters between planets.

Interestingly, the pericenter argument of planet $m_{1}$ shows no signs of being affected by the Lidov-Kozai cycles during most of the evolution, including the phase when its inclination increased. A detailed analysis of this system shows that the eccentricity/inclination excitation occurred in this system mainly due to the effect of close encounters between planets, rather than to the Lidov-Kozai (or secular) effects.

A second case of orbit evolution is shown in Figure 10, and corresponds to Pop-I hot Jupiter on a prograde orbit. In this case, the planet that became hot Jupiter was originally the outermost body in the system (denoted by $m_{2}$ ).

The orbital evolution of all planets is characterized by a prolonged phase of chaotic evolution, when planets move on crossing orbits and interact with each other. During the later stages, the inclinations of $m_{2}$ and $m_{3}$ are periodically excited to values close to $50^{\circ}$, returning to lower values each time the eccentricity suffers a jump. This anti-correlation of $e$ and $I$ is characteristic of the Lidov-Kozai resonance, but is also observed in the circulation domain of $\omega$ in the secular three-body problem.

The evolution of $\omega$ shows some evidence of temporary capture in the Lidov-Kozai resonance (when either $\omega_{3}=90^{\circ}$ or $\omega_{3}=270^{\circ}$; see the bottom panel in Fig. 10), but overall this seems to have only a minor cumulative effect on the final inclination. Pericenter argument rapidly precesses during the very late stage (after $t \sim 1.4 \times 10^{5} \mathrm{yr}$ ) due to the combined effects of tides, relativity and stellar oblateness.

Finally, Figure 11 shows an example of a system that produced the Pop-II hot Jupiter. Here the outer planet $\left(m_{3}\right)$ is ejected from the system at $t \simeq 10^{5}$ years. The system evolves more regularly after this time. Planet $m_{1}$ becomes trapped in the Lidov-Kozai resonance, and remains in the resonance until the semimajor axis drops to very small values. The 
orbital inclination shows no significant long-term evolution in the Lidov-Kozai resonance remaining close to the value attained immediately after the escape of $m_{3}$.

A similar analysis of a large sample of our simulations indicates an important difference between the runs resulting in Pop-I planets, and those generating Pop-II planets. In the first case, due to the fact that all planets remain in the system for the duration of the run, the dynamical evolution shows a prolonged phase of strong interaction between planets, leading to the excitation of their eccentricities and inclinations. Since planetary orbits suffer very strong perturbations, hot Jupiter can form only if the orbital pericenter drops to very small values, where it can be tidally trapped. Consequently, practically all hot Jupiters that formed in these systems belong to Pop I. Also, the system's strong chaoticity produces a larger spread in the inclination distribution, including numerous retrograde orbits in Pop I.

Another consequence of strong interaction in these systems is that the Lidov-Kozai resonance can be sustained only for short intervals of time, and does not seem to be important for the formation of hot Jupiters (retrograde or not). Indeed we found no clear examples of the standard Kozai migration (Wu \& Murray 2003).

It seems instead that the high eccentricities and inclinations are attained due to effects of secular resonances between pairs of planets. We thus agree with Wu \& Lithwick (2011) that the dynamical process responsible for the formation of hot Jupiters is not simply the Kozai resonance, but rather a sort of secular chaos in the regime of high eccentricities $\&$ inclinations. The exact relative importance of the secular chaos and effect of close encounters between planets, however, has yet to be established. The close encounters can clearly generate the AMD needed for the secular chaos to operate, thus explaining the initial orbits used by Wu \& Lithwick (2011), or can even be more central to the formation of hot Jupiter, by continuously pumping AMD into the system until $e \sim 1$.

The orbit evolution of Pop-II Jupiters is qualitatively different from that of Pop-I 
Jupiters, but the mechanisms that operate to produce large eccentricities/inclinations are the same. After the 3rd planet's ejection in the systems that produce Pop II, the outer perturber generally has a large semimajor axis. This causes smaller perturbations on and slower evolution of the inner planet orbit, and permits tidal capture at larger pericentric distance. Since the evolution is less chaotic and close encounters less frequent, the excitation of the inclination is modest. The retrograde orbits are therefore more rare in Pop II than in Pop I.

We tested how the results discussed above depend on the tidal model. For example, we switched of the modifications of the equilibrium tidal model described in Section 2 and/or used different values of $Q_{p}^{\prime}$. We then conducted a set of simulations starting from the same initial conditions as above. Although the results of individual runs were sensitive the assumed tidal model, which is expected from the stochasticity of planet scattering, the overall statistical results were very similar to those discussed above. This shows the origin of hot Jupiters is insensitive of details of the tidal interaction.

\subsection{Comparison with observed hot Jupiters}

To compare our results with observations, we should ideally follow each planetary system for the estimated age of its host star. This is not practical, however, because the analysis of thousands of synthetic systems and dozens of different times would be complicated. We opted for a simplified comparison instead. First we continued the orbits of hot Jupiters, using our semi-analytical method, to 1 Gyr, which is a sort of the average age of known hot-Jupiter's parent stars (e.g. Triaud 2011). Unlike in Sections 3.4 and 3.5., however, the hot Jupiters that reached the Roche radius of the star before 1 Gyr were 

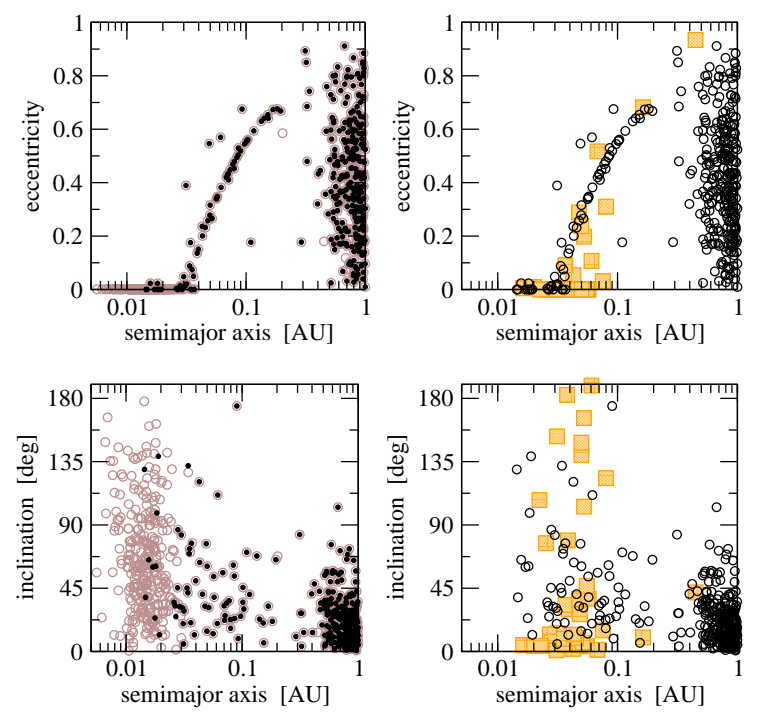

Fig. 12.- Left: Orbit distribution of hot Jupiters that we obtained starting from the 3planet systems. Gray open circles show the transient population of orbits at $10^{8} \mathrm{yr}$, or the moment when the orbit of hot Jupiter became circularized by tides (defined as $e<0.01$ ). Black circles show orbits of planets that survived the tidal decay for $T=10^{9} \mathrm{yrs}$, regardless of their eccentricity. Right: Comparison between the synthetic population (black circles) and known hot Jupiters with measured $|\lambda|$ (orange squares).

removed 2 The final distribution that we obtained here should therefore be characteristic of 'aged' planetary systems.

Results are shown in the left-hand plots of Figure 12, where gray open circles reproduce the data shown in Figure 7, while the black filled circles show only those planets that survive at 1 Gyr. Notably, most hot Jupiters in Pop I disappear as they evolve by tides and are engulfed by the star. Conversely, the orbit distribution of Pop-II Jupiters does not change much. While Population-I hot Jupiters should therefore be expected only around

\footnotetext{
${ }^{2}$ Recall that previously the orbital evolution was stopped when the eccentricity reached $e=0.01$, independently of the timescale when this occurred.
} 
young stars, the Population II is more permanent.

The right-hand plots in Figure 12 show the orbit distribution of planets at 1 Gyr (open black circles), while the orange squares show that of the known hot Jupiters. The distribution of semimajor axes and eccentricities of hot Jupiters produced in our simulations closely matches observations. There is a clear trend, both in our results and observations, that hot Jupiters with larger semimajor axes tend to have larger eccentricities. We discussed this in Section 2.4.

In our simulations, the correlation between final semimajor axes and eccentricities appear as a well defined curve, while the real hot Jupiters seem to be more spread on both sides. This spread may be produced by uncertainties in the estimation of planetary eccentricities, or by a spread of $Q_{p}^{\prime}$ values of real exoplanets. Also, with the Population I now completely removed, our simulations show no hot Jupiters with $a<0.015$ AU, exactly as observed.

For real planets we assumed that the measured $\lambda$ can be interpreted as the orbital tilt, and plot it together with the orbital inclinations of hot Jupiters obtained in our simulations. Note $\lambda$ is the projected misalignment angle; a small value of $\lambda$ therefore does not guarantee that star's spin vector and planet's orbit normal are actually aligned 3

The distributions of both $I$ and $|\lambda|$ are broad, covering the whole range from 0 to $180^{\circ}$, and roughly similar (Figure 12, bottom right panel), which is encouraging. There are several, potentially important differences between these two distributions as well.

\footnotetext{
${ }^{3}$ To compare simulations and observations more precisely, we would need to generate orbit normal vectors having an inclination distribution that our model predicts, and random orientation of nodes. This distribution should then be projected to the observer plane, and compared with observed distributions of $\lambda$ (e.g., Fabrycky \& Winn 2009).
} 
For example, the inclination distribution of surviving hot Jupiters that we obtain in our simulations shows the vast majority of prograde orbits, while the measured values of $\lambda$ indicate that a relatively large fraction $(\sim 20 \%)$ of hot Jupiters are retrograde. Interestingly, as we discuss in the following section, this problem may be resolved if the planetary systems had more than three planets initially (i.e., before scattering).

\section{Four planet systems}

Here we consider the planetary systems with four planets initially. We used the method described in Section 3.3 to set up the initial orbits and masses of four planets. Specifically, the masses of planets were chosen according to Eq. (13), with an additional restraint that

the lightest planet has mass $>0.4 \mathrm{M}_{\mathrm{Jup}}$. In total, 2166 planetary systems were followed. Of these, 498 produced hot Jupiters (as defined by final $a<0.1 \mathrm{AU}$ ), including both Pop I and Pop II. This is $23 \%$, a fraction twice as high as the one obtained with the three planet systems.

The eccentricity and inclination distribution of exoplanets is shown in Figure 13, where we have already separated cases according to the final number of planets in each system. These results were obtained by numerically integrating the systems for $T=10^{8} \mathrm{yr}$, and extending these simulations to $T=10^{9}$ yr using our semi-analytical model. We show the final orbits of all planets at $T=10^{9} \mathrm{yr}$ or, if hot Jupiter reached $e<0.01$, we show the orbits at that time instant.

As in the 3-planet simulations discussed above, we find two distinct populations of hot Jupiters: Population I with $a \leq 0.03 \mathrm{AU}$, and Population II with $a>0.03 \mathrm{AU}$. As before, the Pop-II planets form in the systems that end up with two planets (two planets are ejected in this case), while planet ejection was less common in those systems that produced 

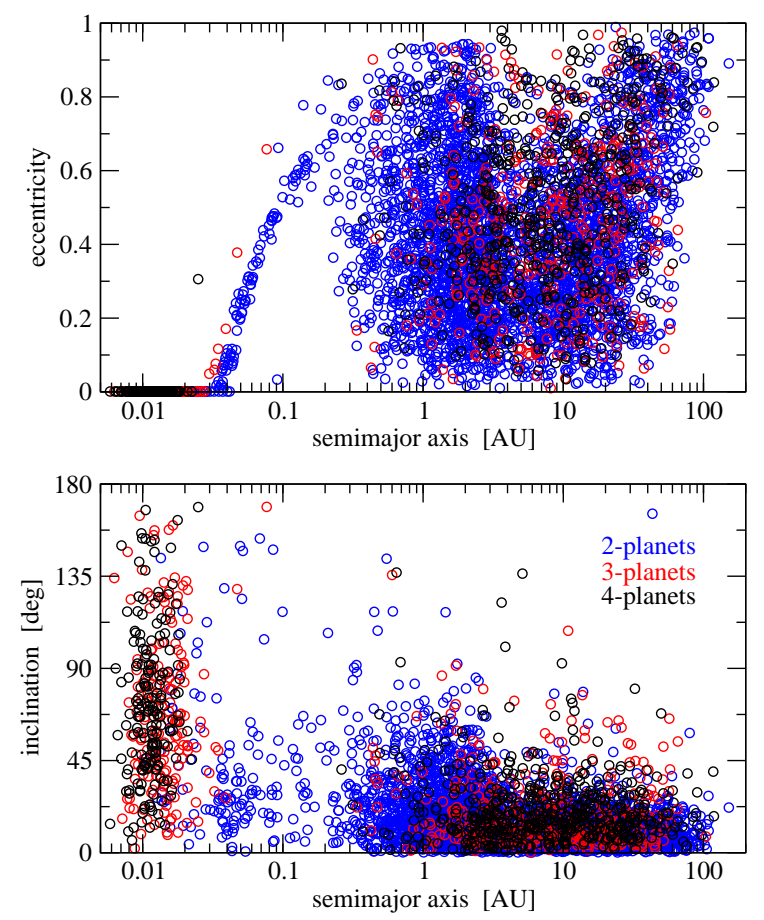

Fig. 13. - Eccentricity (top) and inclination (bottom) distribution of planetary orbits resulting from our 4-planet simulations. Color indicates the number of planets surviving in each system (i.e., blue denotes systems in which two of the initial four planets were ejected, and two survived). Here we stopped the simulation if hot Jupiter formed and acquired a nearly circular orbit (defined as $e<0.01$ ).

Pop I. Interestingly, however, the inclination distribution of Pop-II hot Jupiters is now broader and includes a larger fraction of retrograde orbits than in the 3-planet case.

A detailed analysis of individual 4-planet simulations shows similar mechanisms at work as for the 3-planet systems (see Section 3.5). The orbit evolutions are complex, show a dominant effect of planetary encounters and slow secular interactions that are typically not related to the Lidov-Kozai resonance.

Finally, the left-hand panels in Figure 14 show the final orbits of hot Jupiters at 1 Gyr. This result confirms those obtained with the 3-planet systems. The Pop-I hot Jupiters 

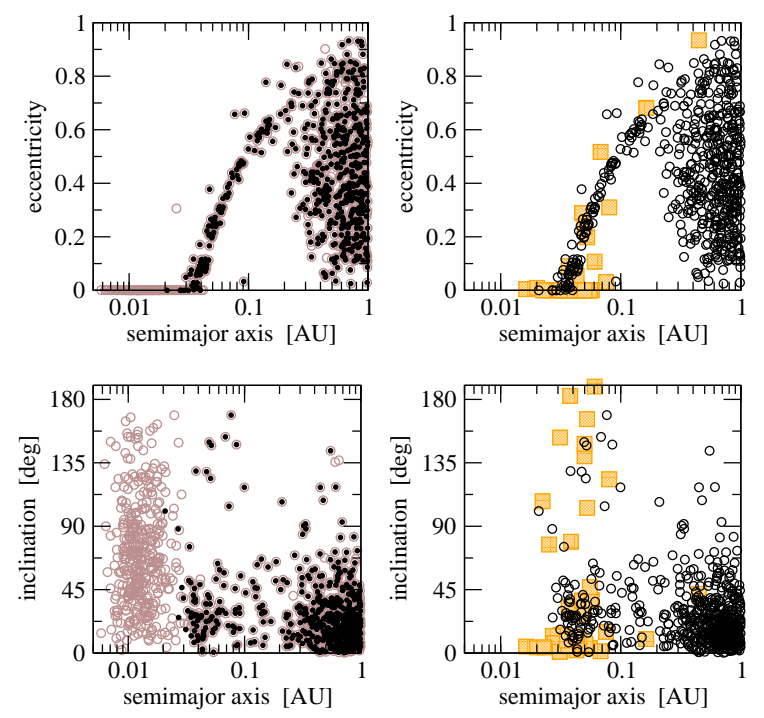

Fig. 14. - Same as Figure 12, but for our four-planet simulations described in Section 4.

do not generally survive, while the orbits in Pop II do not change much at late stages. Both the semimajor axis and eccentricity distribution of final orbits show a good match to observations. The match to the observed 3-day-period pile-up of hot Jupiters is particularly good.

Unlike in the three planet case, the inclination distribution obtained with four initial planets shows a relatively large fraction of retrograde orbits $(\sim 10 \%)$. Also, some of the retrograde orbits now have $I>150^{\circ}$, as required to match several known systems with $|\lambda| \gtrsim 150^{\circ}$. For comparison, observations indicate that $\sim 20 \%$ of hot Jupiters have $|\lambda|>90^{\circ}$. It is not clear at this point whether the difference between simulated $10 \%$ and observed $20 \%$ is significant, mainly because the observational statistics is still pretty low.

\section{Conclusions}

Here we reported a series of simulations in which we followed the orbital and spin evolution of planetary systems starting with three and four planets. The planets were 
initially placed in resonant orbits as expected from their formation and migration in the protoplanetary gas disk. The instability was triggered in each of these systems by breaking the resonant locks. Soon after the onset of instability, planets scatter each other and typically obtain large orbital eccentricities and inclinations. In addition to the gravitational interactions between planets, we also included the effects of relativity, stellar oblateness and tides, which are mainly important for orbits with low pericenter distance. We modified the standard equilibrium model to mimic the effects of dynamical tides for $e \sim 1$.

We found that scattering and subsequent slow secular interaction between planets generate a number of planets in quasi-parabolic orbits that tidally evolve. A fraction of these planets can survive over Gyr timescales. The surviving population provides a good match to observations, including the 3-day-period pile-up of hot Jupiters, nearly circular orbits of hot Jupiter for $\lesssim 0.03 \mathrm{AU}$, correlation between $a$ and $e$ for orbits with larger $a$, etc.

Contrary to previous works, we found the the Kozai resonance is not the dominant evolution path leading to the formation of hot Jupiters. The vast majority of hot Jupiters in our simulations acquire small pericentric distances (and in some cases retrograde orbits) by being scattered by other planets and/or during the subsequent phase of slow secular evolution (typically unrelated to the Kozai resonance).

We find that $\approx 10 \%$ of planetary systems starting with three planets produce hot Jupiters, while this ratio increases to $\approx 23 \%$ if four planets are considered. However, most of these are eliminated by subsequent tidal decay for timescales of the order of 1 Gyr. The proportion of surviving hot planets drop to $\approx 2 \%$ for 3 -planet systems and $\approx 5 \%$ for our 4-planet runs.

In both cases, we find that hot Jupiters can be divided into two populations. The transient Population-I hot Jupiters typically form in the systems where no (in the 3 planet case) or up to one planet (in the 4 planet case) is ejected, have $a<0.03 \mathrm{AU}$, and a very 
broad inclination distribution, including a large fraction of retrograde orbits. These planets continue to evolve tidally and generally do not survive 1 Gyr. They are expected to be present only around younger stars.

The Population-II hot Jupiters form in the systems where one (in the 3 planet case) or two planets are ejected (in the 4 planet case), have $a>0.03 \mathrm{AU}$, and a smaller fraction of retrograde orbits. The Pop-II hot Jupiters are far enough from the central star to have only minimal tidal evolution, and generally survive on Gyr-long time scales. They provide the best match to the observed population of hot Jupiters.

We found that the initial systems with more than three planets tend to produce hot Jupiters more often than the systems with three initial planets, and the orbits of hot Jupiters born in the former systems tend to have broader inclination distribution, including about $10 \%$ of retrograde orbits. Both these characteristics appear to provide a better match to observations (although much work remains to be done). This may suggest that planetary systems emerging from the protoplanetary disks, or at least the ones that seed hot Jupiters, are initially unexpectedly rich in the number of planets.

Acknowledgments: This work has been supported by NSF AAG. C.B. would like to express his gratitude to the Southwest Research Institute and all its staff for invaluable help during the development of this work. 


\section{REFERENCES}

Benitez-Llambay, P., Masset, F., Beaugé, C. 2011. A\&A, 528, A2.

Beutler, G. 2005. "Methods of Celestial Mechanics", Springer-Verlag, Germany.

Chambers, J.E. 1999. MNRAS, 304, 793.

Chatterjee, S., Ford, E.B., Matsumura, S., Rasio, F.A. 2008. ApJ, 686, 580.

Darwin, G.H. 1879. The Observatory, 3, 79.

Deleuil, M. et al. 2011. A\&A, submitted (astroph-1109.3203).

Fabrycky, D.C., Winn, J.N. 2009. ApJ, 696, 1230.

Gladman, B., 1993. Icarus, 106, 247.

Hut, P. 1981. A\&A, 99, 126.

Ivanov, P.B., Papaloizou, J.C.B. 2004. MNRAS, 347, 437.

Ivanov, P.B., Papaloizou, J.C.B. 2007. MNRAS, 376, 682.

Ivanov, P.B., Papaloizou, J.C.B. 2011. CeMDA, 111, 51.

Jackson, B., Greenberg, R., Barnes, R. 2008. ApJ, 678, 1396.

Juric, M., Tremaine, S. 2008. ApJ, 686, 603.

Kley, W. 2003. CeMDA, 87, 85.

Kozai, Y. 1962. AJ, 67, 591.

Lai, D. 1997. ApJ, 490, 847.

Lai, D., Foucart, F., Lin, D.N.C. 2011. MNRAS, 412, 2790. 
Lainey, V., Arlot, J,-E, Karatekin, Ö, Van Hoolst, T. 2009. Nature, 459, 957.

Laskar, J., Robutel, P. 1995. CeMDA, 62, 193.

Levison, H.F., Duncan, M.J. (2000). ApJ, 120, 2117.

Libert, A.-S., Tsiganis, K. 2011a. MNRAS, 412, 2353.

Libert, A.-S., Tsiganis, K. 2011b. CeMDA 111, 201.

Lidov, M.L. 1961. Isk. Sput. Zemli, 8, 119.

Marchal, C., Bozis, G. 1982. CeMDA, 26, 311.

Mardling, R.A. 2007. MNRAS, 382, 1768.

Marzari, F., Weidenschilling. S.J. 2002. Icarus, 156, 570.

Masset, F. A\&ASS, 141, 165.

McArthur, B.E., Benedict, G.F., Barnes, R., Martioli, E., Korzennik, S., Nelan, E., Butler, R.P. 2010. ApJ, 715, 1203.

Moeckel, N., Throop, H.B. 2009. ApJ, 707, 268.

Mignard, F. 1979. Moon and Planets, 20, 301.

Mignard, F. 1980. Moon and Planets, 23, 185.

Morbidelli, A., Tsiganis. K., Crida, A., Levison, H.F., Gomes, R. 2007. AJ, 134, 1790.

Moutou, C., et al., 2011. A\&A, 533, A113.

Nagasawa, M., Ida, S., Bessho, T. 2008. ApJ, 678, 498.

Naoz, S., Farr, W.M., Lithwick, Y., Rasio, F.A., Teyssandier, J. 2011. Nature, 473, 187. 
Pierens, A., Nelson, R.P. 2008. A\&A, 482, 333.

Schlaufman, K.C. 2010. ApJ, 719, 602.

Skumanich, A. 1972, ApJ, 171, 565

Snellgrove, M.D., Papaloizou, J.C.B., Nelson, R.P. 2001. A\&A, 374, 1092.

Thommes, E.W., Bryden, G., Wu, Y., Rasio, F.A. 2008. ApJ, 675, 1538.

Triaud, A.H.M.J. 2011. A\&A, submitted (astroph 1109.5813v1).

Throop, H.B., Bally, J. 2008. AJ, 135, 2380.

Wu, Y., Murray, N. 2003. ApJ, 589, 605.

Wu, Y., Lithwick, Y. 2011. ApJ, 735, 109. 